\title{
Controlled growth of hexagonal GaN pyramids by hot-wall MOCVD
}

\author{
Anders Lundskog, Chih-Wei Hsu, K Fredrik Karlsson, Urban Forsberg, \\ Per-Olof Holtz and Erik Janzén
}

\section{Linköping University Post Print}

N.B.: When citing this work, cite the original article.

Original Publication:

Anders Lundskog, Chih-Wei Hsu, K Fredrik Karlsson, Urban Forsberg, Per-Olof Holtz and Erik Janzén, Controlled growth of hexagonal GaN pyramids by hot-wall MOCVD, 2013, Journal of Crystal Growth, (363), 287-293.

http://dx.doi.org/10.1016/j.jcrysgro.2012.11.014

Copyright: Elsevier

http://www.elsevier.com/

Postprint available at: Linköping University Electronic Press

http://urn.kb.se/resolve?urn=urn:nbn:se:liu:diva-79317 


\section{Controlled growth evolution of hexagonal GaN pyramids grown by hot-wall MOCVD}

Anders Lundskog, Chih-Wei Hsu, Urban Forsberg, Per-Olof Holtz and Erik Janzèn

\section{Abstract}

The growth evolution of hexagonal GaN pyramids are investigated under various growth conditions. The hexagonal GaN pyramids were grown by hot-wall metal organic chemical vapor deposition process (hot-wall MOCVD) on a (0001) oriented GaN template. We concluded the growth of the hexagonal GaN pyramids can be divided into two different regimes defined by the adsorption kinetics of the $\{1101\}$ surfaces of the pyramids. In the adsorption -regime, the hexagonal GaN pyramids expands in $<1101>$-direction. In the zero adsorption -regime, there are no expansion in the $<1101>-$ direction thus the growth ceases after the top (0001) surface is eliminated. The growth regime selection has large impact on the pyramid to pyramid uniformity and the growth evolution of the pyramids. The pyramid to pyramid uniformity was found to rapidly degrade with increasing growthtime in the absorption regime, while no uniformity degradation was observed in the zero adsorption -regime. Large arrays of hexagonal GaN pyramid with apex radiuses less than $3 \mathrm{~nm}$ was achieved in the zero adsorption regime. A simple model is proposed for the uniformity degradation mechanism in the adsorption regime. 


\section{Introduction}

The III-nitrides consisting of $\operatorname{InN}, \mathrm{GaN}$, and AIN have intensely been studied the recent 20 years because of their huge potential for everything from high efficiency solid state lighting $\left[{ }^{1}\right]$ to high frequency power electronics [2]. Most of III-nitrides-based optoelectronic devices reported to date are grown on (0001) oriented sapphire substrates. However, the intrinsic spontaneous and strain induced piezoelectric fields in III-nitrides cause spatial separation of electrons and holes within active quantum wells (QWs) $\left[^{3}\right]$. The spatial separation of the electrons and holes are often referred to as the quantum confined stark effect (QCSE). To diminish the QCSE thinner QWs have generally been employed in the active QWs of LEDs. While the introduction of thinner QWs improves the radiative recombination, it can make carrier injection into the QWs inefficient, possibly resulting in overall lower internal quantum efficiency [3]. The QCSE of QWs drastically reduce when the QWs are grown on semi-polar directions of the GaN crystal $\left.\left.\left[{ }^{4}\right]\right]^{5}\right]$. Semi-polar surfaces may be synthesized by substrate cleavage or selective area growth (SAG) on (0001) oriented substrates. GaN hexagonal pyramids $\left[{ }^{6}\right]\left[{ }^{7}\right]$ and nanowires $\left[{ }^{8}\right]\left[^{9}\right]$ with visible $\{1 \underline{1} 01\},(0001)$, and $\{1 \underline{1} 00\}$ surfaces grown on (0001) oriented substrates have earlier been demonstrated by SAG in by several growth techniques such as metal organic chemical vapor deposition (MOCVD) [] and molecular beam epitaxy (MBE) $\left[{ }^{10}\right]$. The apex of SAG hexagonal GaN pyramids have also been experimentally demonstrated to act as a preferred nucleation site for InGaN quantum dots (QDs) $\left[{ }^{11}\right]\left[{ }^{12}\right]\left[{ }^{13}\right]\left[{ }^{14}\right]$. We have recently shown that the nucleation of InGaN QDs at the apex of the pyramid directly is affected by the geometric properties of the preceding pyramid apex $\left[{ }^{15}\right]$. Hence controlling the preceding apex shape, and size are crucial for any type of InGaN QD optimization process.

In this paper we have studied the growth evolution of hexagonal GaN pyramids on (0001) oriented GaN templates. We have concluded the absorption kinetics of the $\{1 \underline{1} 01\}$ surfaces is detrimental for uniformity optimization. We particularly stress on the pyramid to pyramid uniformity for the reason that it is an important parameter for any type of device application. A qualitative simple model for the absorption kinetics of the $\{1 \underline{1} 01\}$ surface was employed. 


\section{Experimental details}

A $2.0 \mu \mathrm{m}$ thick (0001) oriented $\mathrm{GaN}$ epitaxial film grown on a 4H-SiC substrate starting with a mono crystalline AIN nucleation layer (100 nm thick) was used as substrate for the hexagonal GaN pyramids. The template was grown by a hot-wall MOCVD -process. Detailed information about the growth process can be found elsewhere $\left[{ }^{16}\right]\left[{ }^{17}\right]$. Ammonia (NH3), trimethylgallium (TMGa), and trimethylaluminum (TMAI) were used as precursors. A mixture of purified hydrogen $(\mathrm{H} 2)$ and nitrogen (N2) was used as carrier gas. The relative H2/N2 -ratio was set to 2.1, where a N2 flow of 9 SLM (standard liters per minute) where employed. On top of the template structure, a $30 \mathrm{~nm}$ thick amorphous $\mathrm{SiN}_{\mathrm{x}}$ film was grown by external plasma enhanced CVD (PECVD) -process. The hexagonal GaN pyramids were formed by regrowth in the same hot-wall MOCVD reactor through a rectangular array of circular lithographically patterned SiN holes created by a UV-lithography/RIA-etching processes. The NH3 and TMGa flow was locked to 6 SLM and 3.3 SCCM during the hexagonal GaN pyramid growth unless else are specifically written.

Growth was performed on two different mask layouts. Both mask layouts consisted of $25 * 25$ holes repeated in a square array across the substrate. On the first mask layout, the mask fill factor (defined as the open area / (mask + open area) -ratio) was 0.67. To avoid coalescence of the pyramids during longer runs, a relatively small fill factor of 0.33 was used on the second mask layout. The SiN window opening diameter was 3.0, and $6.0 \mu \mathrm{m}$ on mask layout number 1 , and 3.0,6.0, and $10.0 \mu \mathrm{m}$ on mask layout number 2. From here on are pyramids emerging from 3.0, 6.0, and $10.0 \mu \mathrm{m} \mathrm{SiN} \mathrm{window}$ openings simply referred to as $3.0,6.0$, and $10.0 \mu \mathrm{m}$ pyramids respectively.

The dimensions of the hexagonal GaN pyramids were determined by scanning electron microscopy (SEM) and analyzed by a MATLAB image toolbox script. To obtain accurate values of the pyramid dimensions, we averaged the dimension of 10 to 12 independent hexagonal GaN pyramids located in a close vicinity of each other. The following measurement entities were introduced; $A_{t}$ as the (0001) truncation area in $\mu \mathrm{m}^{2}$ at the pyramid apex and $S_{b}$ as the base dimension in $\mu \mathrm{m}$ of one facet next to the SiN film. The relevant dimensions are illustrated in figure 1 . As each hexagonal pyramid poses six facets, each measured individual value of $S_{b}$ presented is an average of 60 to 72 measured entities. In a similar fashion is the non-uniformity (defined as the standard deviation / mean value) of $S_{b}$, calculated from 60 to 72 pyramid facets originating from 10-12 independent hexagonal GaN pyramids.

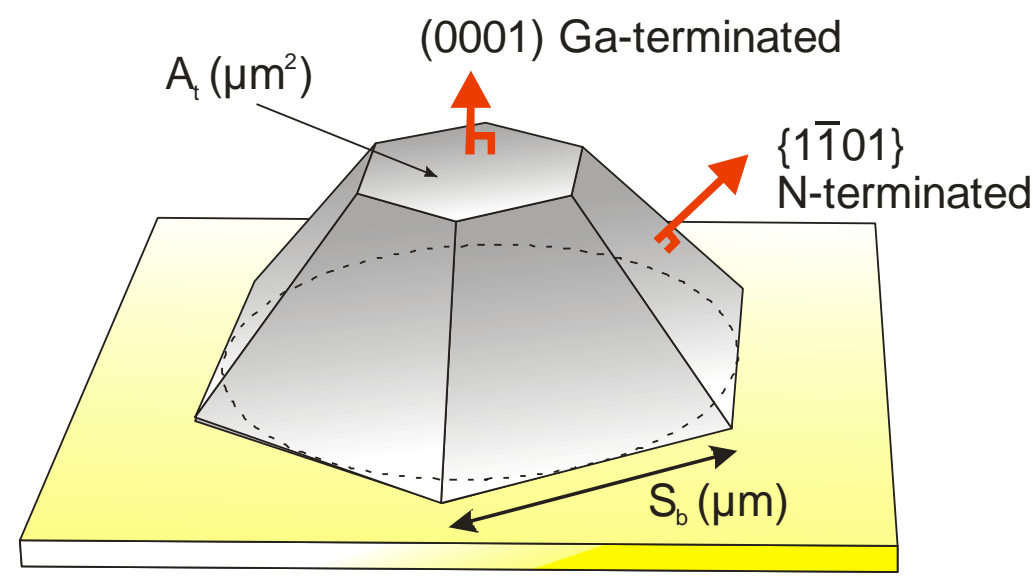

Figure 1: Measurement entities of the hexagonal GaN pyramid. The red arrows correspond to surface normal of the respective GaN surfaces. 


\section{Results: Low temperature growth of hexagonal GaN pyramids}

In the first part of the study the growth temperature was locked at $1000{ }^{\circ} \mathrm{C}$. In figure 2 a) and b) the characteristic dimensions $S_{b}$ and $A_{t}$ versus the growthtime are plotted. Each data point in figure 2 is an individual run. From figure 2 a) $S_{b}$ increases at a constant rate with increasing growthtime for both 3.0 and $6.0 \mu \mathrm{m}$ pyramids. By a simple geometric conversion factor a growthrate of $1.2 \mu \mathrm{m} / \mathrm{h}$ of the $\{1 \underline{101}\}$ surfaces can be calculated from the slope if figure 2 a) for both 3.0 and $6.0 \mu \mathrm{m}$ pyramids. In figure 2 b) $A_{t}$ do not converge at $0 \mu \mathrm{m} 2$ with increasing growthtime i.e. the top area of the pyramid never disappears completely. This is a consequence of the self-limited facet growth mechanism which been observed in both MOCVD grown tetragonal GaAs $\left[{ }^{18}\right]\left[{ }^{19}\right]$ and hexagonal GaN $\left[{ }^{20}\right]-$ pyramids. The self-limited facet growth mechanism is explained as follows: At the initial stage of the growth, the (0001) GaN surface becomes narrower with increasing growth time as the surrounding $\{1101\}$ surfaces expand in an in-plane direction. At some point, the lateral size of (0001) area, $A_{t}$, of the pyramid is comparable with the diffusion length of the arriving adatoms. Adatoms on the (0001) surface therefore out-diffuse from the (0001) surface to the $\{1 \underline{101\}}$ surfaces where the sticking probabilities of the adatoms are lower compared to the (0001) surface. If the out-diffused adatoms stick to the neighboring $\{1 \underline{1} 01\} \mathrm{GaN}$-surface the pyramid expands latterly (i.e. $A_{t}$ increases). Eventually a balanced in and out -diffusion of adatoms at the apex will be reached, resulting in a constant $A_{t}$ with increasing growthtime.
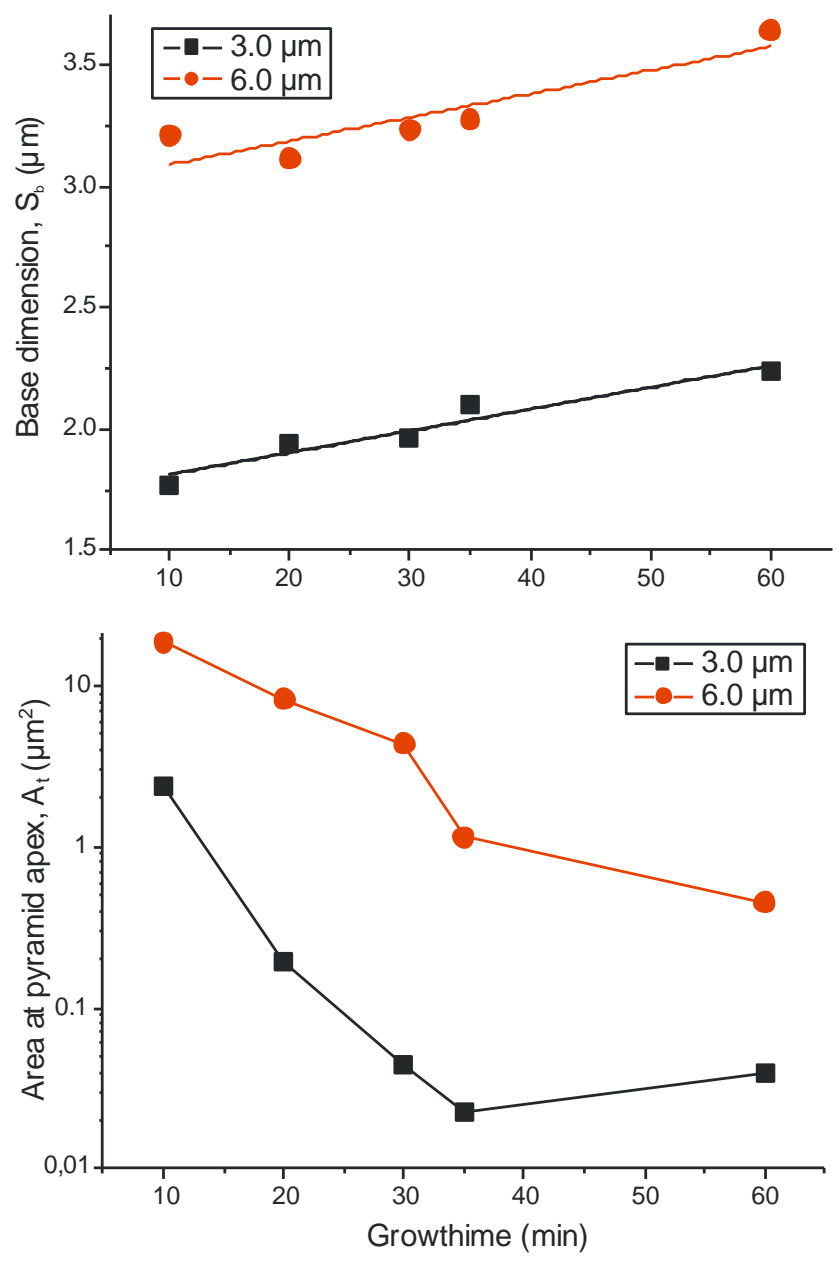

Figure 2: Characteristic mean dimensions of hexagonal GaN pyramids versus growthtime. From the slope of figure 2 a) the growthrate of the $\{1101\}$ surface was calculated. The growth temperature was set to $1000 \mathrm{C}$. 


\section{Results: High temperature growth of hexagonal GaN pyramids}

In the second part of this paper the growth temperature was set to $1060^{\circ} \mathrm{C}$. In figure $3 \mathrm{SEM}$ pictures of three selected 3.0, 6.0 and $10 \mu \mathrm{m}$ hexagonal GaN pyramids from with mask layout number 2 are shown. The hexagonal GaN pyramids in figure 2) are overgrown i.e. after 30 minutes of growth; the sample was taken out, characterized by SEM, and put back in the growth chamber for regrowth. The timescale shown in figure 2 is the total accumulated growth time. After 30 minutes of growth, highly uniform and esthetically attractive hexagonal pyramids emerged from $3.0 \mu \mathrm{m}$ SiN window opening diameters. In contrast to the growth performed at $1000{ }^{\circ} \mathrm{C}$ no visible (0001) truncation area could be observed $\left(A_{t}=0 \mu \mathrm{m}^{2}\right)$. The appearance and size of the $3.0 \mu \mathrm{m}$ hexagonal GaN pyramids did not change with increasing growth time. Furthermore, cross sectional SEM on one of the $3.0 \mu \mathrm{m}$ pyramids are shown in figure $3 \mathrm{~b}$ ). The radius of the very tip was determined to be less than $3 \mathrm{~nm}$. The apex size of the tip is close to the ultimate resolution of the SEM hence the actual tip radius could be smaller. In figure 3 a) a planar view SEM of the pyramid in figure 3 b) are shown.

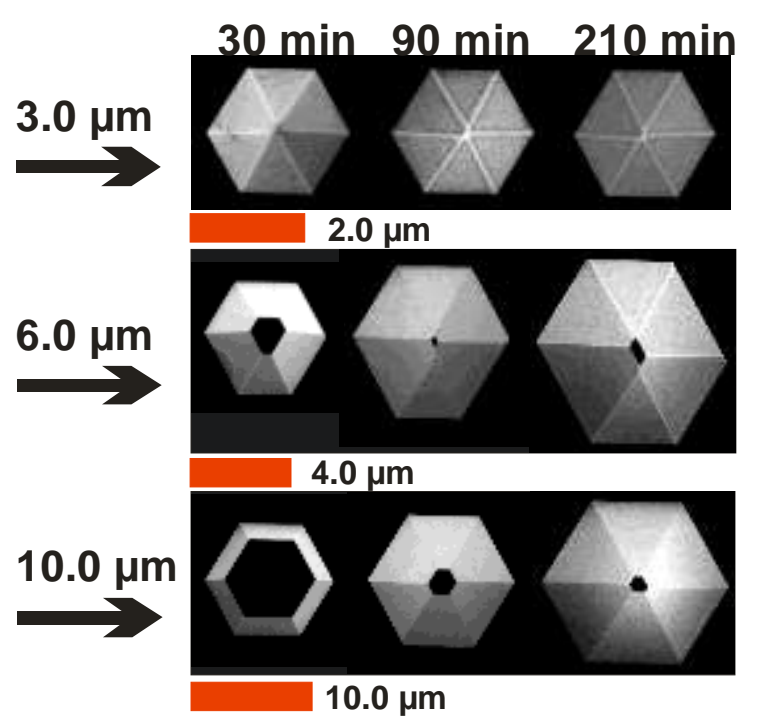

Figure 2: Overgrown hexagonal GaN pyramids from mask layout with SiN window openings. The growth of the hexagonal GaN pyramids of each column was grown during the same experiment.
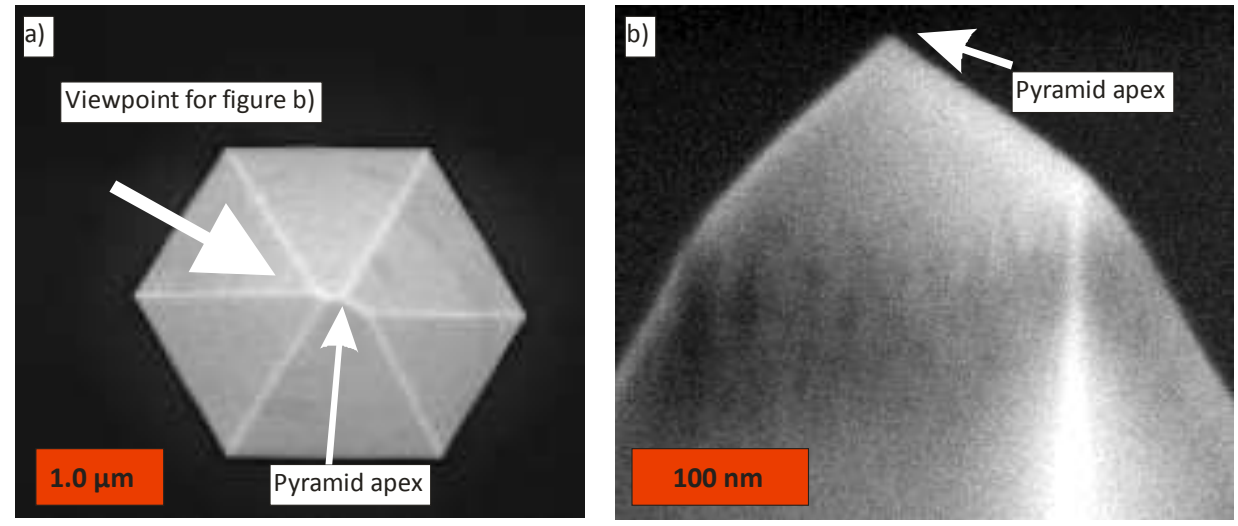

Figure 3: SEM picture of one hexagonal GaN pyramid grown at $1060^{\circ} \mathrm{C}$ for 30 minutes. In a) view from above and b) cross sectional view. The pyramid apex radius in the picture is roughly $3 \mathrm{~nm}$. 
In figure $4 \mathrm{a}$ ) and b) the characteristic dimensions $S_{b}$ and $A_{t}$ versus the growthtime from mask layout number 1 and 2 are respectively shown. The inset of figure 4 a) shows the base dimension $S_{b}$ versus the growthtime on mask layout number 2. By the use of a simple geometric factor and the slope of figure 4 a) the growthrate of the $\{1 \underline{1} 01\}$ surfaces can be calculated. The growth rate on the $\{1101\}$ surfaces was to our surprise dependent of the SiN mask opening diameter. We underline that the arrays consisting of $25 * 25$ window openings was scaled in a 1:1 relation (leaving fill factors constant) for the 3.0,6.0, and $10 \mu \mathrm{m}$ pyramids. The $\{1 \underline{101}\}$ growth rate are $0 \mu \mathrm{m} / \mathrm{h}$ on $3.0 \mu \mathrm{m}$ openings on both mask layout 1 and 2 , and 0.23 and $0.375 \mu \mathrm{m} / \mathrm{h}$ on $6.0 \mu \mathrm{m}$ pyramids on mask 1 and 2 respectively. The growthrate discrepancy between the two investigated masks, are due to enhanced source supply as a consequence of the decreased fill factor. The growth rate of the $\{1 \underline{101}\}$ surface on $10.0 \mu \mathrm{m}$ openings was determined to be $0.55 \mu \mathrm{m} / \mathrm{h}$. In figure $4 \mathrm{~b}$ ) $A_{t}$ versus growthtime are shown. The 3.0 $\mu \mathrm{m}$ pyramids closed after roughly 30 minutes of growth leaving nominally sharp hexagonal pyramids (as shown in figure 2 a). On the 6.0 and $10.0 \mu \mathrm{m}$ the apex region never closed after extended growth time of 210 minutes (as shown in figure $2 b$ and $c$ ).
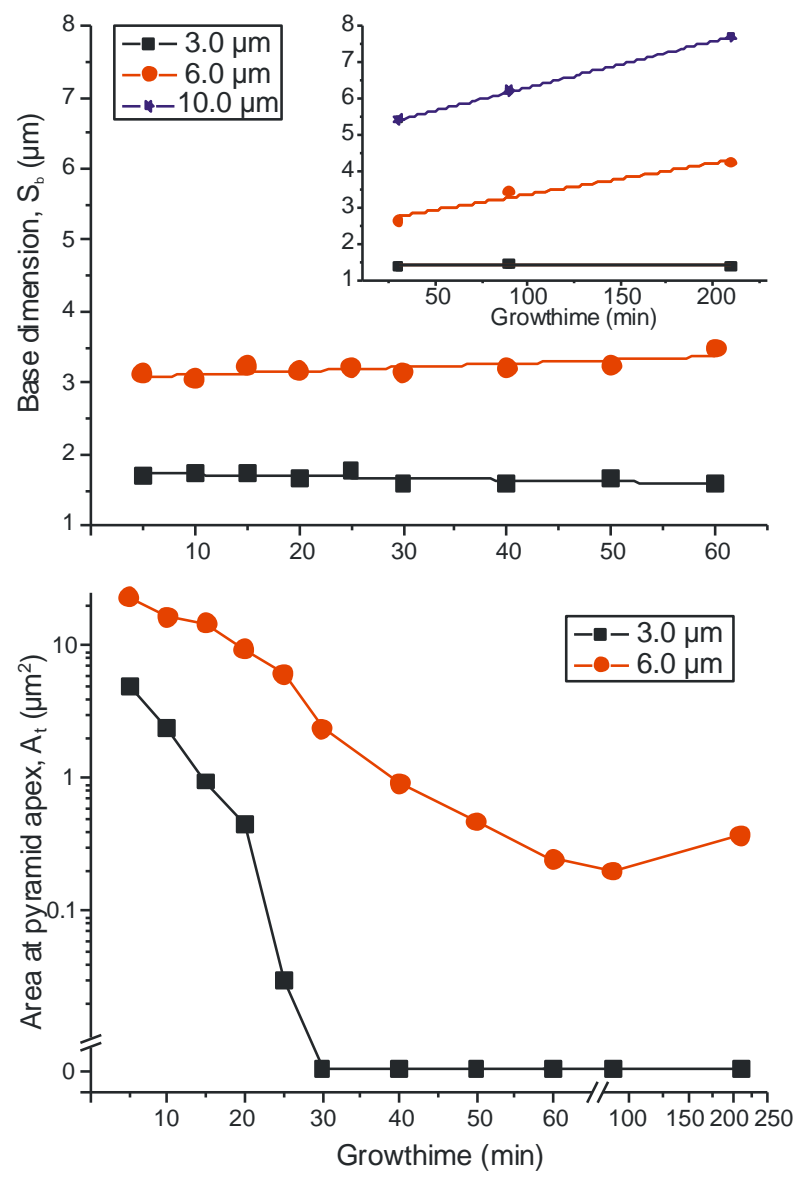

Figure 4: Characteristic dimensions, $S_{b}$ and $A_{t}$ of hexagonal GaN pyramids versus the growthtime. The inset of figure 4a shows pyramid regrowth from mask layout number 2. In Figure 6 b) the curves corresponding to 3.0 and $6.0 \mu \mathrm{m}$ were extended with the regrown pyramids from mask layout number 2.

\section{Discussion and growth model}

In MOCVD growth of GaN, the growthrate is generally determined by the competition between adsorption and desorption of adatoms from the vapor phase. In our experiments the arrival rate of adatoms are near constant (due to fixed TMGa flow) while desorption rate increases exponentially 
with the growth temperature [] (material is assumed to be in its equilibrium state). In the low temperature regime $\left(1000^{\circ} \mathrm{C}\right)$, the adatom desorption rate are small in comparison to the arrival rate of new adatoms, thus the $\{1101\}$-surface growthrate are larger than $0 \mu \mathrm{m} / \mathrm{h}$. At $1060{ }^{\circ} \mathrm{C}$ the adsorption and desorption on the $\{1101\}$ are equal in magnitude, resulting in a $\{1101\}$ growthrate of $0 \mu \mathrm{m} / \mathrm{h}$. Under these particular growth conditions the pyramid still grows in the $<0001>$-direction. Inevitably a sharp apex will ultimately form. As soon as a sharp apex is formed, a "status quo" occurs, and the pyramid appearance does not change with increasing growthtime (as shown in figure 2 a). We emphasize that this particular "status quo" growth window can be shifted by growth temperature and TMGa flow. A $\{1101\}$ surface growthrate of $0 \mu \mathrm{m} / \mathrm{h}$ was achieved at growth temperatures of $1045{ }^{\circ} \mathrm{C}$, and $1085{ }^{\circ} \mathrm{C}$ with TMGa flow rates of 1.9 , and $5.7 \mathrm{SCCM}$ respectively. The results fit well to a simple kinetic model from reference $\left[{ }^{21}\right]$ where the growth rate $\left(R_{g}\right)$ equals:

$$
\left.R_{g}=\beta_{V}\left(\frac{P}{P_{e}(T)}-1\right)\right)
$$

were $\mathrm{P}$ is the partial pressure of gallium containing adatoms in vapor phase, $P_{e}(T)$ the equilibrium vapor pressure of $\mathrm{GaN}$, and $\beta_{V}$ a kinetic coefficient. The kinetic coefficient contains for example the surface roughness in terms of probability of finding a kink, the activation energy for lattice incorporation, and growth temperature. The equilibrium vapor pressure of $G a N, P_{e}(T)$, is an exponentially increasing function of the temperature [] hence the most sensitive parameter. A similar expression for the growth rate without the kinetic coefficient was developed more than 100 years ago $\left[{ }^{22}\right]\left[{ }^{23}\right]$.

From these results we classify the growth of hexagonal GaN pyramids in two regimes by the adsorption and desorption kinetics of the $\{1101\}$ surfaces. At low temperature (or high partial pressure of TMGa) the adsorption of adatoms on the $\{1 \underline{101\}}$ results in lateral (0001) in-plane expansion of the pyramid thus the self limited facet growth stops the hexagonal GaN pyramid from forming a sharp apex. If the temperature is increased (or low partial pressure of TMGa) till the absorption and desorption rates of the $\{1 \underline{1} 01\}$ surface are balanced, thus no lateral expansion of the pyramid occurs. However, the pyramid still grows in the $<0001>$ direction and eventually will sharp apex form.

The suggested model does not explain the $\{1 \underline{1} 01\}$ surface growthrate dependence on the SiN window opening diameter. However, we have noticed that the growthrate of the $6.0 \mu \mathrm{m}$ pyramids also follow equation 2 but with a small temperature and/or TMGa flow -offset as sharp pyramid apexes and \{1101\} surface growth rate of $0 \mu \mathrm{m} / \mathrm{h}$ was realized on $6.0 \mu \mathrm{m}$ pyramids at $1070 \mathrm{C}$ with a TMGa flow of $3.3 \mathrm{SCCM}$. We believe the size dependence of the $\{1 \underline{101}\}$ growth rate is due to the chemistry differences over the substrate, as the temperature variations over the substrate are too small to affect the nucleation $\left.{ }^{24}\right]$.

\section{Results: Uniformity degradation}

In the next section the growth regime selection impact on the pyramid to pyramid uniformity (from here on we will refer to the non-uniformity) will be discussed. A low magnification SEM picture of 3.0 $\mu \mathrm{m}$ pyramids grown in the zero adsorption regime is shown in figure 5 . The non-uniformity of $S_{b}$ is less than $3 \%$ and the apex sharpness is estimated to be as the apex shown in figure 3 . In figure 6 a) the non-uniformity of $S_{b}$ versus the growthtime is shown. The non-uniformity nearly constant for all 
individual runs at roughly $5 \%$ which we believe are caused by deviations in SiN window opening diameters. Figure 5 is an excellent example of how SAG can be used for ultimate control of 3Dnanostructures.

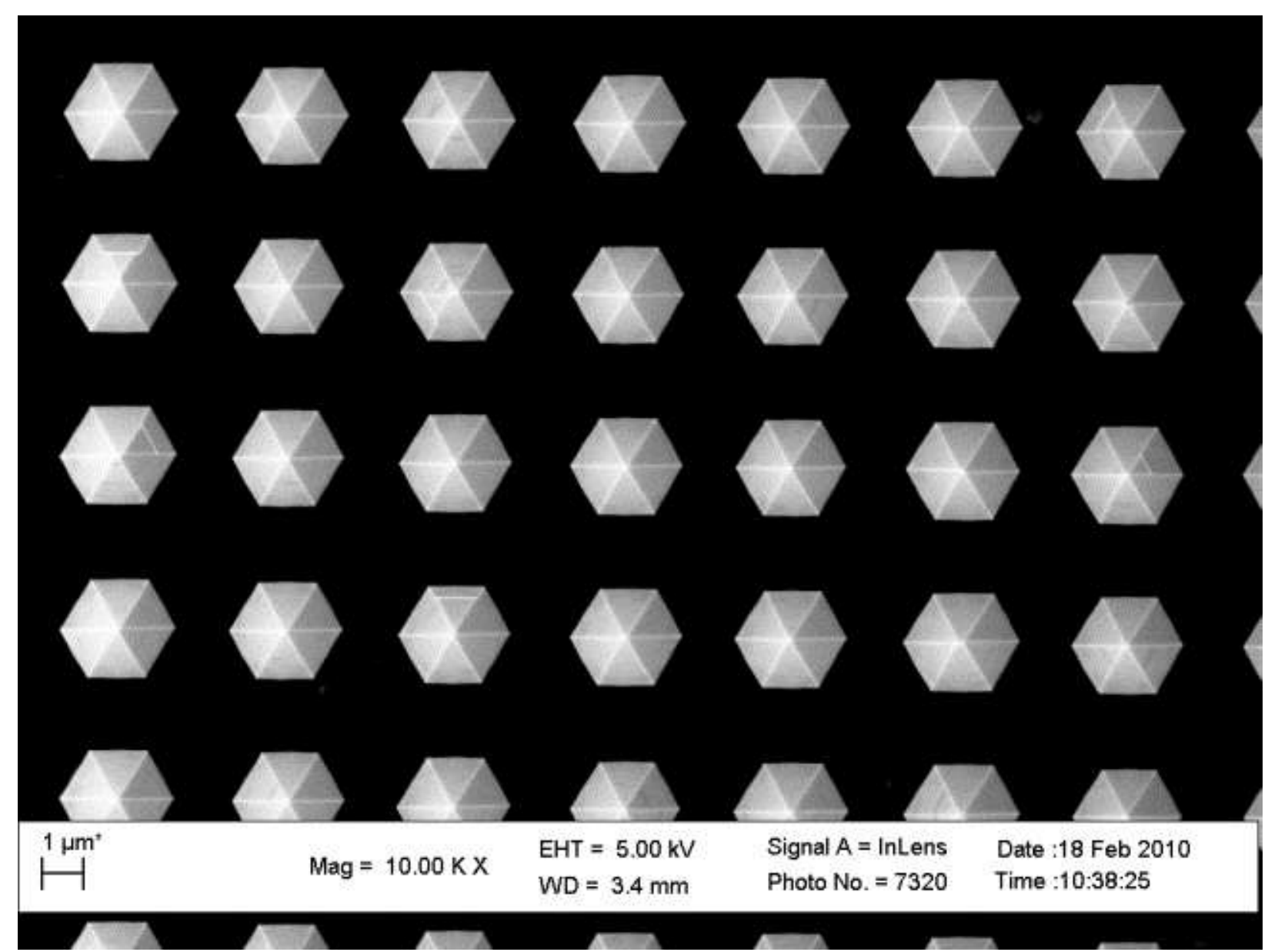

Figure 5: Low magnification SEM of hexagonal GaN pyramids illustrating the excellent uniformity achieved in the zeroadsorption regime. The pyramids on this picture are slightly more densely packed compared to the ones in figure 2, 4 and 6.

In figure $6 \mathrm{a}$ ) and b) the non-uniformity of $S_{B}$ versus growth time at growth temperatures of 1060, and $1000{ }^{\circ} \mathrm{C}$ are respectively shown. The $6.0 \mu \mathrm{m}$ pyramids grown at both 1060 and $1000{ }^{\circ} \mathrm{C}$ are according to our growth model grown in the adsorption regime. Here, a sudden increase (from $2 \%$ to $10 \%$ ) of the non-uniformity occurs after roughly 40 and 30 minutes of growth for $6.0 \mu \mathrm{m}$ pyramids at 1060 and $1000{ }^{\circ} \mathrm{C}$ respectively. The sudden uniformity degradation is illustrated in figure $6 \mathrm{c}$ ) and d). The $3.0 \mu \mathrm{m}$ pyramids grown at $1000^{\circ} \mathrm{C}$ on the other hand, increase in a near linear relation.

The non-uniformity increase of $S_{b}$ for $6.0 \mu \mathrm{m}$ pyramids grown at 1000 and $1060{ }^{\circ} \mathrm{C}$ occur roughly as the truncation area approaches $1.0 \mu \mathrm{m} 2$ (compare with figure $2 \mathrm{~b}$ ) and $4 \mathrm{~b}$ )). This indicates that the degradation of the uniformity occurs as the self-limited facet mechanism starts. Furthermore are vertical $\{1 \underline{100}\}$ surfaces are exposed as the self-limited facet mechanism starts. On $6.0 \mu \mathrm{m}$ pyramids for example, clear $\{1 \underline{100}\}$ surfaces are visible when the growthtime are set to 50 minutes or more. The $\{1 \underline{1} 00\}$ surfaces on a pyramid are visualized in figure $8 \mathrm{f}$ ). Simultaneously as the self-limited facet mechanism starts, the apex region of the pyramid skews. After 50 minutes of growth the hexagonal shaped apexes are rearranged to irregular triangular/tetragonal/pentagonal. In figure 8 e) a SEM picture of a triangular apex is shown. 

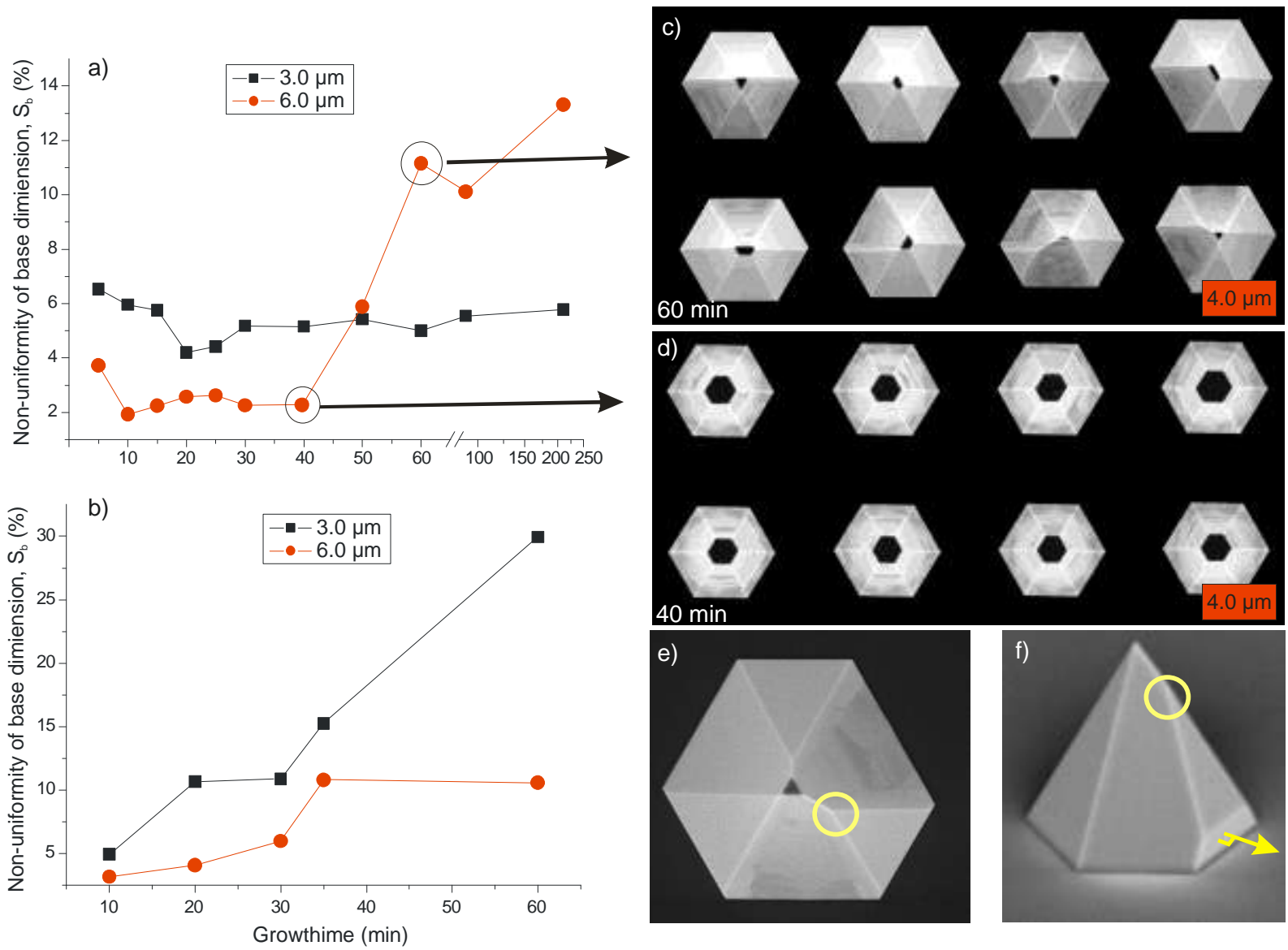

Figure 6: Non-uniformity of pyramid base dimension $S_{b}$ versus growthtime for pyramids grown at a) $1060 \mathrm{C}$ and b) $1000 \mathrm{C}$. SEM pictures of $6.0 \mathrm{~mm}$ hexagonal GaN pyramid arrays grown at $1060 \mathrm{C}$ for c) $10 \mathrm{~min}$, d) $40 \mathrm{~min}$, and e) $60 \mathrm{~min}$. The red scalebar in figure 8 e) applies to all figures. The datapoints from 5-60 minutes in figure 8 a) correspond to individual runs from mask number 1 while data points at 90, and 210 minutes are results from overgrown sample at mask number 2 .

\section{Discussion: Uniformity degradation}

In MOCVD growth, the carrier gas selection plays an important role in determination of post-growth visible surfaces. It was recently demonstrated that hydrogen, selectively etches gallium face $\{1 \underline{1} 0 \underline{1}\}$ surfaces to $\{1 \underline{1} 00\}$ surfaces in hydrogen rich environments. In reference $\left[{ }^{25}\right]\left[\left[^{26}\right]\left[{ }^{27}\right]\right.$ it were recently suggested preferential etching from $\{1 \underline{1} 01\}$ to $\{1 \underline{1} 00\}$ surfaces where the formation mechanism of nanowires. In this study the H2/N2 ratio was fixed to 2.1 during the entire growth. However, $\{1100\}$ surfaces were only reveled in the later part of the growth. Hence, it is unlikely the $\{1 \underline{100}\}$ surfaces where reveled due to hydrogen etching $(\{1 \underline{1} 00\}$ surfaces would otherwise be visible on $3.0 \mu \mathrm{m}$ pyramids grown for $210 \mathrm{~min}$ at $1060 \mathrm{C}$ ).

We believe the uniformity degradation of the pyramids is due to the surface energy minimization processes the pyramids endure during the growth. According to Wulff the equilibrium shape of the grown crystal is obtained by minimizing the total surface energy subjected to the restriction of constant volume $\left[{ }^{28}\right]$. In the case of hexagonal GaN pyramids the growthrate of the (0001) surface is much larger compared to the $\{1 \underline{101}\}$ and $\{1 \underline{100}\}$ surfaces, thus it is reasonable to assume the crystal is trying to remove the (0001) due to its relatively high surface energy. However, as the self-limited facet growth mechanism hinders the removal of the (0001) surface in the adsorption regime, the pyramid desperately finds alternative ways for (0001) surface area reduction. The area of a hexagonal shaped apex is momentary written as $A_{1}(t)=6 \sqrt{3}\left(R_{g} \cdot t\right)^{2}$ where $R_{g}$ is the planar 
projection of the $\{1 \underline{101}\}$-growthrate, and $t$ the time. In a similar way, the area of triangular shaped apex is momentary written as $A_{2}(t)=4 / \sqrt{3} \cdot\left(R_{g} \cdot t\right)^{2}$. The ratio between $A_{1}(t) / A_{2}(t)$ equals $2 / 9$; hence rearranging a hexagonal apex to a triangular is a more efficient way to reduce the (0001) surface area. The different apex layouts are illustrated in figure 7 a) and b). However, simple rearrangement of the pyramid apex does not conserve the angles between the pyramid sidewalls. To maintain stable $\{1 \underline{1} 01\}$ surface the pyramid crossing of two $\{1 \underline{1} 01\}$ surfaces "slides down" forming a small ridge. The $\{1 \underline{101}\}$ surface crossing is highlighted with a yellow circle in figure 6 e) and f). The rearrangement from hexagonal to triangular apexes and sliding down of the crossing points between two $\{1 \underline{101\}}$ surface does not imply the appearance of $\{1 \underline{100}\}$ surfaces. Therefore we suspect the $\{1 \underline{100}\}$ surfaces reveals as the crystal approach its equilibrium shape.
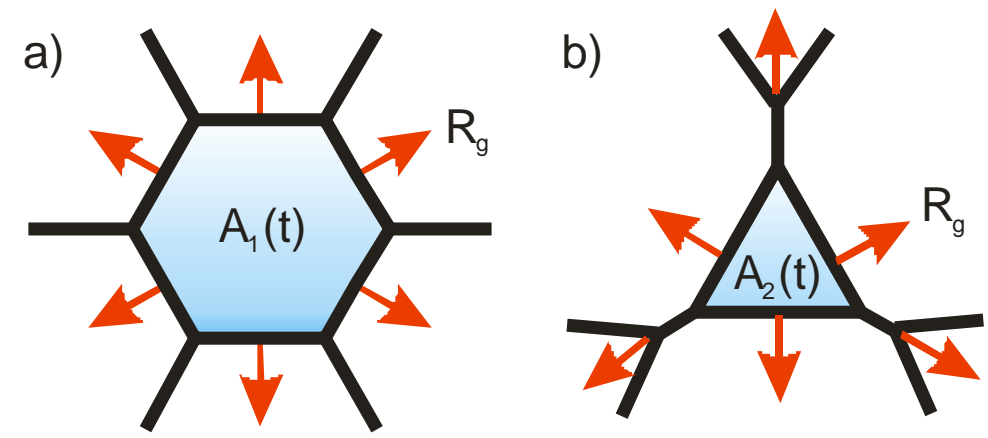

Figure 7: Momentary expansion of the (0001) truncation area of a hexagonal GaN pyramid. The apex arrangement is in figure a) hexagonal and b) triangular. The red arrows correspond to a constant growthrate. A1 and A2 are the area of the truncation area at any time $t$.

The proposed model also explains why larger pyramids typically are less sensitive to uniformity degradation in comparison to smaller ones. In this study a maximum non-uniformity of approximately 30,13, and $9 \%$ for 3.0, 6.0, and $10.0 \mu \mathrm{m}$ pyramids were respectively observed. Accordingly, this is due to the fact the relative energy gain are much larger for small pyramids compared to larger ones.

\section{Summary}

In summary, we have developed basic understanding for controlled growth of (0001) -oriented hexagonal GaN pyramids by hot wall MOCVD. The growth was divided in two regimes dependent of the adsorption kinetics of the $\{1 \underline{101}\}$ surface. In the zero adsorption regime highly uniform large arrays of hexagonal GaN pyramids with apex radiuses less than $3 \mathrm{~nm}$ was achieved. In the adsorption regime the uniformity of the pyramids quickly degraded as the apexes were near completion. A simple model was proposed to explain the uniformity degradation mechanism.

\section{Acknowledgements}

This work was supported by the NANO-N consortium funded by the Swedish Foundation for Strategic Research.

\section{References}

\footnotetext{
${ }^{1}$ Siddha Pimputkar, Nature Photonics 3, 180 - 182 (2009) doi:10.1038/nphoton.2009.32
} 
${ }^{2}$ Dirk Wiegner, International Journal of Microwave and Wireless Technologies (2010), 2: 95 DOI: 10.1017/S175907871000022X

${ }^{3}$ Jae-Hyun Ryou, IEEE JOURNAL OF SELECTED TOPICS IN QUANTUM ELECTRONICS, VOL. 15, NO. 4, JULY/AUGUST 2009 DOI: 10.1109/JSTQE.2009.2014170

${ }^{4}$ Tetsuya Takeuchi , Jpn. J. Appl. Phys. 39 (2000) pp. 413-416 DOI: 10.1143/JJAP.39.413

${ }^{5}$ A. E. Romanov J. Appl. Phys. 100, 023522 (2006); doi: 10.1063/1.2218385

${ }^{6}$ Kazumasa Hiramatsu 2001 J. Phys.: Condens. Matter 136961 doi:10.1088/0953-8984/13/32/306

${ }^{7}$ B. Beaumont, physica status solidi (b) Volume 227, Issue 1, pages 1-43, September 2001 DOI: 10.1002/1521-3951(200109)227:1<1::AID-PSSB1>3.0.CO;2-Q

${ }^{8}$ S. F. Li Cryst. Growth Des., 2011, 11 (5), pp 1573-1577 DOI: 10.1021/cg101537m

${ }^{9}$ Stephen D. Hersee, Nano Lett., 2006, 6 (8), pp 1808-1811 DOI: 10.1021/nl060553t

${ }^{10}$ Hiroto Sekiguchi, Phys. Status Solidi C 7, No. 10, 2374-2377 (2010) / DOI 10.1002/pssc.201083915

${ }^{11}$ V. Pérez-Solórzano, Appl. Phys. Lett. 87, 163121 (2005) ; doi:10.1063/1.2108126

${ }^{12}$ P. R. Edwards, Appl. Phys. Lett. 85, 4281 (2004) ; doi:10.1063/1.1815043

${ }^{13}$ Koichi Tachibana, Appl. Phys. Lett. 76, 3212 (2000) ; doi:10.1063/1.126632

${ }^{14}$ Chih-Wei Hsu, Nano Lett., 2011, 11 (6), pp 2415-2418; doi: 10.1021/nl200810v

${ }^{15}$ Letter från oss

${ }^{16}$ U. Forsberg, Journal of Crystal Growth Volume 311, Issue 10, 1 May 2009, Pages 3007-3010; doi:10.1016/j.jcrysgro.2009.01.045

${ }^{17}$ A. Kakanakova-Georgieva Cryst. Growth Des., 2009, 9 (2), pp 880-884; doi: 10.1021/cg8005663

${ }^{18}$ Seigo Ando Jpn. J. Appl. Phys. 32 (1993) pp. L104-L106; DOI: 10.1143/JJAP.32.L104

${ }^{19}$ Kazuhide Kumakura, Jpn. J. Appl. Phys. 34 (1995) pp. 4387-4389, DOI: 10.1143/JJAP.34.4387

${ }^{20}$ Shota Kitamura, Japanese Journal of Applied Physics, Volume 34, Issue 9B, pp. L1184-L1186 (1995). DOI: 10.1143/JJAP.34.L1184

${ }^{21}$ Ivan V Marko, Crystal Growth for Beginners, second edition, page 189, ISBN-10: 9812382453

${ }^{22}$ Knudsen, M. (1909), Annalen der Physik, 334: 179-193. doi: 10.1002/andp.19093340614

${ }^{23}$ Hertz, H. (1882) Annalen der Physik, 253: 177-193. doi: 10.1002/andp.18822531002

${ }^{24}$ Conclusion based on steady state thermodynamic simulation in COMSOL of the entire growth cell. 
${ }^{25}$ W. Bergbauer, Journal of Crystal Growth Volume 315, Issue 1, 15 January 2011, Pages 164167 doi:10.1016/j.jcrysgro.2010.07.067

${ }^{26}$ Shunfeng Li, Phys. Status Solidi C 8, No. 7-8, 2318-2320 (2011) / DOI 10.1002/pssc.201001003

${ }^{27}$ W Bergbauer et al 2010 Nanotechnology 21305201 doi:10.1088/0957-4484/21/30/305201

${ }^{28}$ Ivan V Marko, Crystal Growth for Beginners, second edition, page 18 ISBN-10: 9812382453 